\title{
BILATERAL PRIMARY NON-HODGKIN'S LYMPHOMA OF BREAST. A RARE ENTITY
}

Narayan Das

1. Assistant Professor. Department of General Surgery, Agartala Government Medical College \& G. B. Pant Hospital Agartala. Tripura.

\section{CORRESPONDING AUTHOR:}

Dr. Narayan Das, (Gen. Surgery), FIAGES,

Old Kalibari lane, (Near Skylark Club),

Kerchoumohani, Krishnanagar,

Agartala, Tripura (West) - 799001.

E-mail: narayandas9862019118@gmail.com

ABSTRACT: Primary lymphoma of the breast is rare. Bilateral involvement is more rare. This report describes the case of 14 year female patient presenting with bilateral breast's lump diagnosed on histopathological examination as primary Non-Hodgkin's lymphoma.

KEY WORDS: Breast, Primary Bilateral Non-Hodgkin's lymphoma.

INTRODUCTION: Primary lymphoma of the breast is rare . Bilateral involvement of the breast is rarer (1). It comprises $0.04 \%$ to $0.05 \%$ of all primary malignant tumour of the breast and $1.7 \%$ to $2.2 \%$ of all extranodal lymphoma (2). This report documents a case of 14 year old female patient presenting with bilateral breast's lump without any axillary mass. Histopathological examination of excised breast's lump diagnosed as primary Non-Hodgkin's lymphoma.

CASE REPORT: A 14 year old female patient presented with chief complaint of a palpable mass in her each breast for one month duration. Physical examination revealed a $5 \mathrm{~cm} \mathrm{X} 5 \mathrm{~cm}$, welldemarcated mobile tender mass in her each breast. There were no palpable nodes in either axilla. Her past medical history was unremarkable, and family history was negative for breast malignancies. Mammography showed a large mass of her each breast with multiple opacities in its contest. Ultrasonography of breast confirmed the present of a hypervascularized non homogeneous mass with ill defined margin in each breast. The patient was referred for fine needle aspiration cytology (FNAC) from the both breast's lump. During aspiration, adequate blood stained material was obtained but result was negative for malignant cells. Considering the clinical diagnosis of breast lump, excision of lumps were done under GA, with adequate monitoring and vigilance (Fig. 1,2). Histopathological examination of excised breast's lumps indicates Non-Hodgkin's lymphoma (Fig.3, 4). The patient was referred to oncologist for further plan of management.

DISCUSSION: Primary lymphoma of the breast is a rare entity . It constitute $0.04 \%$ to $0.05 \%$ of all primary malignant tumours of the breast. Bilateral Non-Hodgkins lymphoma of the breast is a rare tumour(1). The criteria for diagnosis of primary breast lymphoma include (i) The availability of adequate pathology material. (ii) Both mammary tissue and lymphomatous infiltrate present.(iii) No wide spread disease or preceding extramammary lymphoma, and (iv) Homolateral axillary lymph node involvement, which is considered acceptable (2). The age incidence of PBL is highly variable. It occurs between 9 years to 85 years, but frequently has 
been seen in the fifth decade. Bilateral primary Non-Hodgkins has been reported to occur in $1 \%$ to $14 \%$ of cases . The clinical features are short history, rapidly enlarging breast mass, absence of nipple discharge and retraction. "B" symptoms(10\% weight loss, night sweats, fever) are unusual features and laboratory evaluation is usually normal (3). Diagnosis of breast lymphoma almost always requires excisional biopsy or needle aspiration biopsy (4). Many authors reported FNAC is a reliable diagnostic technique, in accordance with the high sensitivity y varying from $83 \%$ to $100 \%$ (5), but in my case, FNAC is not a reliable diagnostic technique. Histopathological examination is very effective tool to really upon for confirmation of diagnosis . Various treatment modalities have been advocated include surgery with radiotherapy or chemotherapy or radiotherapy with chemotherapy or radiotherapy alone (6). Reported 5 year overall survival rates varied from $9 \%$ to $85 \%$.

CONCLUSION Although rare, lymphoma should be considered in the differential diagnosis of a breast's lump. To the best of my knowledge this case is unique in its clinicopathological presentation and also emphasizes the role of excisional biopsy for exact diagnosis of breast's tumour.

\section{REFERENCES:}

1. Freaman C, Berg SW, Catlar SS. Occurrence and prognosis of extranodal lymphoma. Cancer 1972;29:252-60.

2. Wiseman C, Liao KT. Primary lymphoma of the breast. Cancer 1972;29:1705-12.

3. Liu FF, Clark RM. Primary lymphoma of the breast. Clin Radio 1986;37:567-70.

4. Anuradha, Sinha A, Ramrakhiani D. Primary Non-Hodgkin's lymphoma of the breast. Acta Cytologica 2005; 49:661-65.

5. Levine PH, Zamulo R, Yee HT. Role of fine needle aspiration cytology in breast lymphoma.Diag Cytopathol 2004;30:661-65.

6. EL-GhazaryIMH, Singletary SE. Surgical management of primary lymphoma of the breast.Ann Surg 1991;214:724-26.

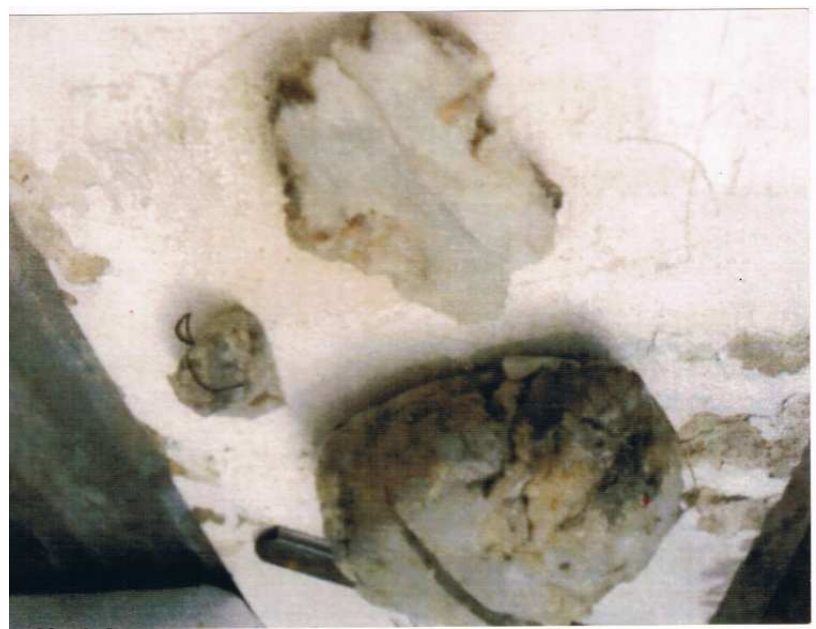

Fig.1-Excised lump of left breast 


\section{CASE REPORT}

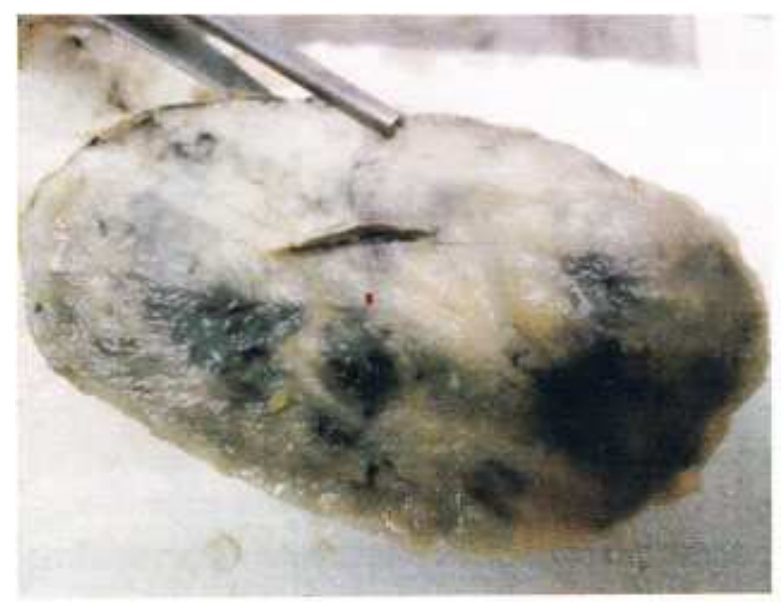

Fig.2-Excised lump of right breast

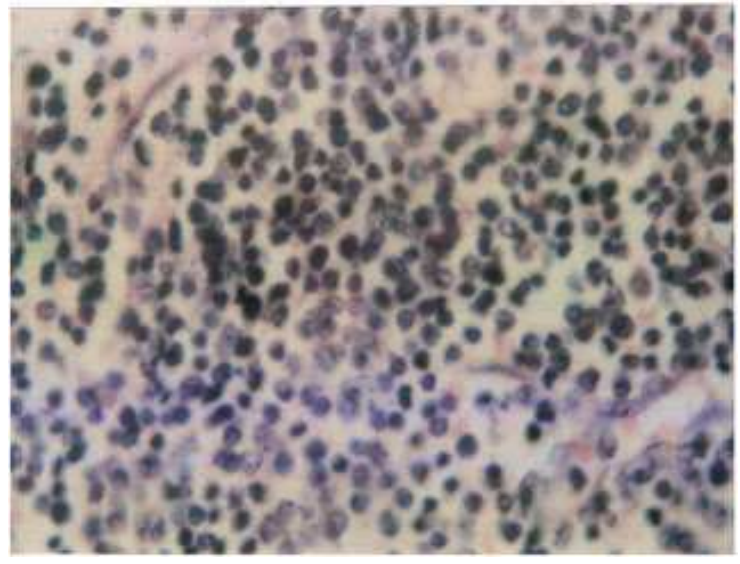

Fig.3-Histopathological examination of left breast 's lump showing features of NonHodgkin's lymphoma.

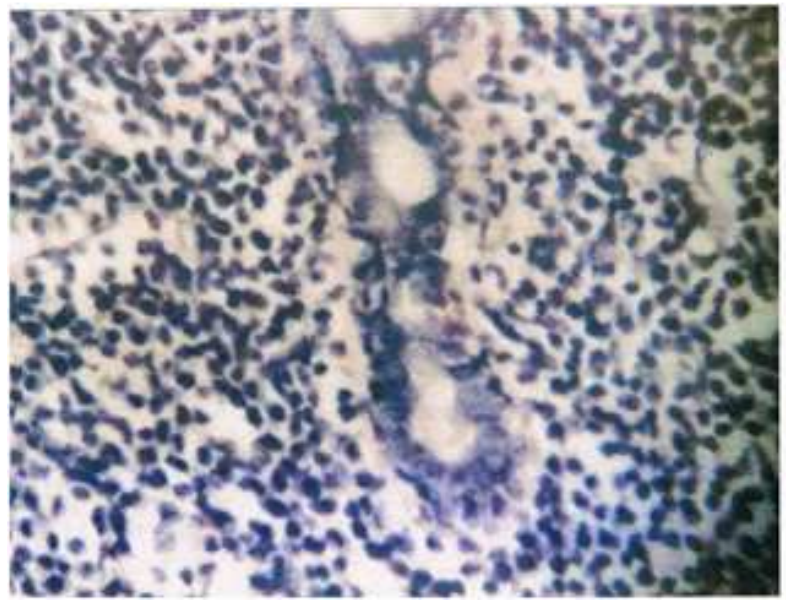

Fig.4-Histopathological examination of right breast 's lump showing features of NonHodgkin's lymphoma. 